\title{
Tracking of The Most Significant Laboratory Parameters For The Identification of Covid-19: An Overview on The Different Blood Tests
}

Seenaa Mohammed Ali

Community Health Department

Technical College of Health

Sulaimani Polytechnic University

Sulaimani, Iraq

seenaa.ali@spu.edu.iq

\begin{tabular}{l} 
Article Info \\
\hline $\begin{array}{l}\text { Special Issue on Coronavirus } \\
\text { (COVID-19) }\end{array}$ \\
DOI: \\
10.24017/covid.13 \\
Article history: \\
Received : 29 May 2020 \\
Accepted : 06 June 2020 \\
Keywords: \\
COVID-19, SARC-COV2, \\
2019-nCoV, Laboratory \\
parameters, Blood test
\end{tabular}

\section{Article Info}

Special Issue on Coronavirus

DOI:

10.24017/covid.13

Article history:

Received : 29 May 2020

Accepted : 06 June 2020

parameters, Blood test

\section{ABSTRACT}

An outbreak of 2019 novel coronavirus disease (COVID-19) began in China during December 2019 which unexpectedly spread to other countries and caused high mortality all over the world. COVID-19 disease primarily manifests as a respiratory tract infection. However, emerging data indicate that it should be regarded as a systemic disease for affecting multiple systems such as cardiovascular, respiratory, gastrointestinal and immune system. There is an accelerated need for detecting the laboratory tests that can aid in identifying infected people and asymptomatic carriers to control the virus transmission process. Although the clinical manifestation of COVID-19 has been widely defined, an overview of the most significant laboratory findings in patients with COVID-19 infection is still limited. Elevation was the predominate result among most of the laboratory parameters while a few decreased in value. Laboratory data have shown that most patients had a decrease in lymphocyte count, Eosinophils count and albumin level. Also, laboratory data recorded an elevation in Leukocyte, ESR, PT, D-dimer, PCT, CRP, ALT, AST, Bilirubin, Creatinine, CK, LDH, Ferritin, Troponin, Myoglobin, IL-6, IL10 and TNF. In general, the parameters had more prominent laboratory abnormalities in severe cases than with non-severe cases. It is well known that laboratory tests results play an important role and can support the early diagnosis of many diseases. This study was carried out to review the abnormalities among the laboratory tests and track the parameters that showed a frequently significant result supporting the primary detection of SARS-COV-2 infection. 


\section{INTRODUCTION}

On 31 Dec 2019, China's heath officials inform WHO about a novel coronavirus that caused a cluster of pneumonia cases [1]. On February 11, 2020, the International Committee on Taxonomy of Viruses (ICTV) declared that this virus was named severe acute respiratory syndrome coronavirus 2 (SARS-CoV-2) [2] while, on the same day, World Health Organization (WHO) named the disease as coronavirus disease COVID-19 [3]. Although the mortality rate of COVID-19 is lower than Middle East Respiratory Syndrome (MERS) and Severe Acute Respiratory Syndrome (SARS), COVID-19 is found to be more severe and can spread in the community more easily than MARS and SARS [4]. The aggressive behavior and highly contagious feature of this novel coronavirus SARS-CoV-2, forced WHO to announce COVID-19 as a pandemic disease on 11/3. In Iraq, the first case was detected in 24 February [5], while the first case of COVID-19 was confirmed in Iraqi Kurdistan_on the first of March [6]. By mid-March, Iraqi health authorities had banned major public events, suspended schools, and closed malls and gathering places. To date (May 29, 2020), the number of infected people all over the world surpasses 5 million [1]. One reason for the rapid spread is that symptoms in the early stage are similar with other pneumonia diseases [7] and it is hard to differentiate it if the patients have atypical symptoms [8]. This increases the difficulty of controlling its transmission, since infected persons can not recognize specific symptoms unless it progresses to a moderate or severe stage [9]. The main problem facing the health care system is the progression of the cases from mild to moderate and severe stages, the late two stages need hospitalized care which also increase the possibility of spreading the virus during visitations or admissions to the hospital.

As recommended by WHO, routine identification of SARS-CoV-2 must be done by the highly specific molecular method RT-PCR. This method is also considered in the assessment of asymptomatic or mildly symptomatic cases [10]. Unfortunately, this advanced technique is limited in many countries and the available materials are not sufficient for all suspected cases. According to that, countries with limited molecular diagnostic capacity have to rely on other laboratory tests that can give primary identification while waiting for the confirmation of RTPCR result (if available). In addition, several recent studies have reported around $20 \%$ falsenegative results for this test [11-13]. These limitations make RT-PCR unsuitable for rapid and large-scale screening. The Chinese Guideline of Diagnosis and treatment of COVID-19 posted that confirmation of COVID-19 infection needs nucleic acid testing of respiratory tract samples. However, clinical diagnosis based on symptoms, exposures, blood tests and chest imaging of suspected cases can be used in the primary identification of suspected cases [14]. Thus, a simple blood test could help in identifying false-positive/negative RT-PCR tests as well as play a useful role in the screening of potential infected individuals with COVID-19 in countries which suffer from a large shortage of RT-PCR reagents and specialized laboratories. A tremendous number of blood test results are accessible since the emergence of COVID-19. Prospective studies had listed many blood test parameters: Complete blood cells count, Leukocyte, Lymphocytes, Neutrophils, Eosinophils, Platelets, ESR, Hemoglobin, PT, Ddimer, PCT, CRP, Albumin, ALT, AST, Bilirubin, Creatinine, CK, LH, Ferritin, Glucose, Troponin, Myoglobin, IL-6, TNF and many others. Most publications are prospective and conducted china [15-29] in while a few studies are published from other countries [30-33]. Lately, a prospective study from Iran has been published [34]. Elevation was predominate result among the blood parameters while a few decreased in value. In general, the parameters had more prominent laboratory abnormalities in severe cases than with non-severe cases. However, a full clinical and laboratory characterization is still required and a better understanding is prerequisite to provide improved guidance [10] .

Till now, there is no standard lab parameters for the identification of the virus. The objective of this study is to summarize the finding of different lab parameters reported in currently available studies and to highlight the ones with a significant result. 


\section{METHODS AND MATERIALS}

PubMed, Scopus, Google Scholar, Elsevier, and Science of direct were searched. Also, the following websites were searched: the WHO and CDC (Centers for Disease Control and Prevention. The search included articles published from January until the end of May 2020. Only peer-reviewed published papers of observational studies that included human patients and was written in English were included in this comprehensive search. Only studies that enrolled patients with COVID-19 infections confirmed by RT-PCR were included in the current study. The search included articles published from January till the end of May 2020.The following terms was used: COVID-19, SARC-COV2, 2019-nCoV, coronavirus, identification, diagnosis, parameters, significant and laboratory.

\subsection{Molecular method RT-PCR}

\section{RESULTS}

WHO mentioned that the nucleic acid amplification tests (NAAT) should be used for screening the virus in suspected cases. A well-known technique such as reverse transcription polymerase chain reaction (RT-PCR) is recommended. So, labs worldwide have customized the test for this novel coronavirus by using different primers for selecting different sections of the virus's genetic sequence. Some countries shared their protocols which can be accessed for the common good [35]. The recent diagnosis protocols for the COVID-19 have been listed in a local study published recently. Most testing for COVID-19 is currently done on viral genetic material from upper respiratory tract specimens; nasopharyngeal swab (NP) or oropharyngeal swab (OP). If the patient develops a productive cough, sample from lower respiratory tract (sputum) should be collected and tested for COVID-19 [36].

\subsection{Serological method IgM/IgG}

In cases where RT-PCR is negative and there is a strong epidemiological relation coto COVID-19 infection, paired serum samples (in the acute and convalescent phase) could support diagnosis [36]. Some studies with COVID-19 serological data successfully detected both IgM/IgG antibodies on the serum of infected patients with SARS-CoV-2 virus [37-41].

This method relies on the detection of specific $\mathrm{IgM} / \mathrm{IgG}$ antibodies as a response to infections which can detect patients at different infection stages. The $\operatorname{IgM}$ antibody can be detected as early as day 3 after exposure.

\subsection{Routine Blood tests}

Through the screening of publications that recorded the findings of different laboratory tests for the identification of the COVID-19, the results of all blood parameters that were mentioned have been collected. These include: Complete blood cells count, Leukocytes, Lymphocytes, Neutrophils, Eosinophils, Platelets, ESR, Hemoglobin, prothrombin time (PT), D-dimer, procalcitonin (PCT), C-reactive protein (CRP), Albumin, alanine aminotransferase (ALT), aspartate aminotransferase (AST), Bilirubin, Creatinine, creatine kinase (CK), lactate dehydrogenase (LDH), Ferritin, Glucose, Troponin, Myoglobin, Interleukin 6 (IL-6), Interleukin 10 and Tumor necrosis factor (TNF). Elevation was the predominate result among most of the blood parameters [15-26], [42].

Laboratory data have shown that most patients had a decrease in lymphocyte count, Eosinophils count, platelets count, hemoglobin, and albumin level. Also, laboratory data recorded an elevation in Leukocyte, ESR, PT, D-dimer, PCT, CRP, ALT, AST, Bilirubin, Creatinine, CK, LDH, Ferritin, Troponin, Myoglobin, IL-6, IL10 and TNF. Among these parameters, a few are frequently encountered in patients with COVID-19 infection which include: Leukocyte, lymphocyte count, ESR, D-dimer, CRP, LDH, PCT, ALT, AST and creatinine. The variation in the decrease or elevation value of these parameters have been noticed in many studies [17-20]. The strong association between the decrease in lymphocyte count, Eosinophil count and albumin has been noticed in patients with the COVID-19 disease. Also, High levels of D-dimer, PCT, LDH, CRP and Ferritin have been observed. Among the scanning parameters, the ones with significant result which are recommended as supportive tests for the identification and monitoring of COVID-19 cases was selected. These significant parameters are: lymphocyte count, eosinophil count, LDH and CRP, which is routinely 
available in all labs and is also mentioned as significant in publications to aid in the primary identification of suspected cases of SARS-COV-2 when the characteristic clinical symptoms are yet to be significant.

\subsection{Significant Hematological parameters}

Complete blood count (CBC) is a cheap and available test currently done by an automated analyzer in advanced labs. Luckily, the conventional method of blood film is available in all labs and the result of the total and differential blood cells count can be achieved by either a hematologist or a lab practitioner. Among hematological parameters, lymphopenia is clearly associated with disease severity. The common result recorded among the CBC parameters in patients with COVID-19 is a decrease in lymphocyte count which can be noticed in the early stage of infection, less commonly mentioned is D-dimer elevation. [19], [20]. Also patients who have died from COVID-19 have had significantly lower lymphocyte counts than survivors. In fact, reduction of lymphocytes may be an important factor for monitoring the severity of the disease [21]. Two studies [22], [23] recorded a significant decline in the number of eosinophil count in patients which can be easily monitored along with lymphocyte count.

\subsection{Significant Biochemical parameters}

Detection of inflammatory factors helps to preliminarily evaluate the immune status of patients. C-reactive protein (CRP) is available, cheap, fast and easy test. The high sensitivity elevated value have been recorded by many studies [24-27]. Therefore, this test will help in the detecting of suspected cases. One study [28] measured the ratio of lymphocyte-to-Creactive protein ratio (LCR) in severe COVID-19 cases as both are inflammation markers that reflect systemic inflammatory response. Also, the result was significant for monitoring the progression of the disease. Among the list of biochemical tests usually presented as a package done by an automated system, a significant elevation in the enzyme Lactate Dehydrogenase $(\mathrm{LDH})$ was noticed in patients with SARS-CoV-2, less commonly mentioned is ALT, AST, and creatine kinase elevation [25], [26], [29], [34]. Fortunately, this test can be done as a single test by a specific kit which is available, cheap, fast and easy.

Interestingly, Chinese diagnosis guidelines used a combination of imaging technique (like chest X-ray or CT scan) finding and specific lab parameter result of white blood cells and lymphocyte counts for the initial identification of suspected cases. The typical early stage chest image features bilateral multiple patchy ground glass opacities [43]. Because it is available, the advanced procedure computed topography (CT scan) was used as a primary tool for the initial identification during the COVID-19 outbreak in China [11], [43], [44].

Fortunately, the same features can be detected (but with less sensitivity) by the conventional plain radiography X-ray [45], [46] which is cheap and available in all hospitals.

According to that, countries with limited molecular diagnostic capacity can depend on other tests that can give primary identification while waiting for the confirmation of RT-PCR result (if available).

\section{DISCUSSION}

According to WHO, the decision of subjecting suspected patients to lab tests should be based on patient's history and clinical symptoms. Although RT-PCR test provides a highly specific result, there are many limitations listed by the CDC guideline [47] such as false negative due to improper collection or insufficient number of virus present in the specimen. Besides, this impractical test requires expensive lab facilities with technical sophistication and restrictive biosafety levels. Also, collection of multiple specimens' types in different period of time from the same patient is advocated, since the optimum specimen types and timing for peak viral levels during infection caused by COVID-19 have not been discovered [44]. Interestingly, many studies proved that chest radiographic findings of the computed topography (CT scan) are more sensitive in comparison with RT-PCR result especially in detecting the infection in the early stages [11], [44], [46]. Recently, CDC announced the development of a serology laboratory test which detects $\operatorname{IgM} / \operatorname{IgG}$ specific antibodies to aid in the determination of 
screening the potentially exposed US population to SARS-CoV-2 virus. On the first of April, the Food and Drug Administration (FDA) Authorized Cellex Inc. to produce the product qSARS-CoV-2 IgG/IgM Rapid Test for the qualitative detection of IgM and IgG antibodies against SARS-CoV-2 in serum, plasm or whole blood of the suspected cases of COVID-19 [41]. With the development of a specific $\operatorname{IgG} / \operatorname{IgM}$ antibody test which can be easily applied in the clinical laboratory of any hospital, detection of patients at different infection stages would be possible. If it became widely available in the future, a fast-preforming, large-scaled serological study can be conducted to collect information about the immunity status of the community. This tests can play a critical role in controlling COVID-19 by helping in identifying people who have previous exposure to the SARS-CoV-2 virus and developed an immune response. Such information can be used to help determine people who are no longer susceptible to infection and can return to their normal life and communicate safely with other people. Although molecular identification of SARS-CoV-2 is the gold-standard for COVID19 diagnosis, common laboratory parameters play a key role in case detection and progression monitoring in developing countries with limited access to molecular diagnostics. According to that, countries with limited molecular diagnostic capacity should depend on other tests that can give primary identification while waiting for the confirmation of RT-PCR result (if available). An early epidemiological study [42] published during the outbreak in China summarized the weekly report from the Chinese Center for Disease Control and Prevention which included 72314 Cases [14]. However, only $62 \%$ of systematic cases were diagnosed based on positive PCR test results. $15 \%$ of cases were diagnosed based on clinical symptoms, exposures, and presence of lung imaging features consistent with coronavirus pneumonia, while $22 \%$ of suspected cases were diagnosed based on clinical symptoms and exposures only. The remaining 1\% represent asymptomatic people diagnosed as COVID-19 cases by PCR. The explanation for this unusual practice is that PCR testing capacity was insufficient at that time. So, clinical diagnosis may be made based on exposures, symptoms, and chest imaging [42]. Another experience in Italy, where the infected cases passed 100,000 with a mortality rate of $10 \%$, the shortage, specialized laboratory and reagents forced the government to limit the swab test to suspected cases who show symptoms of severe acute respiratory syndrome [48]. In this Italian study, the blood test results of 207 patients with COVID19 symptoms admitted to the Hospital emergency room, were tested by RT-PCR. Of them, 102 tested negative whereas 105 tested positive. The levels of white blood cells (WBCs), platelets, alanine aminotransferase (ALT), C-reactive protein (CRP), aspartate aminotransferase (AST), lactate dehydrogenase (LDH) and alkaline phosphatase (ALP) in the two groups have been analyzed, with the aim of highlighting statistically significant differences that could be useful for the identification of negative and positive COVID-19 patients. Different recommendations for using specific lab test can be found in different studies. A systemic review orchestrated by researchers from 14 countries [16] summarize the laboratory findings of 19 researches with 39 case reports. The study revealed the $\mathrm{P}$ value of 12 parameters. Another review done by two Italian authors [17] summarize the lab results of eight Chinese studies which included 22 parameters and the significance of the parameters was showed by percentage. Furthermore, meta-analysis and systematic review of 43 studies mentioned the result of 11 laboratory parameters assessed by $\mathrm{I}^{2}$ value [18]. Limitation of these studies can be recorded because of the variation in the final presentation of the results. Also, not all studies divided the cases as severe and non-severe. In general, the parameters had more prominent laboratory abnormalities in severe cases than with non-severe cases. A few parameters show a decrease in value; Lymphocyte, Eosinophil and LDH which are usually notable in non-severe stages while Albumin and Hemoglobin lowering in severe stage.

\section{CONCLUSION}

- Amplification of viral RNA by RT-PCR serves as the gold standard for confirmation of infection, yet it needs a long turnaround time. Additionally, the need for certified laboratories, expensive equipment and trained personnel caused many countries to limit the RT-PCR tests 
only to individuals with pronounced respiratory syndrome symptoms. So, there is a need for alternative, more accessible and less expensive tests.

- Thus, an available significant blood test could help in identifying false- negative/positive RT-PCR results and also might be used in countries under crises or in countries hurting from a shortage of RT-PCR reagents and/or specialized laboratories as an inexpensive and available alternative test to identify potential COVID-19 patients.

- Elevation was predominate among most of the laboratory parameters while a few were decreased in value. Laboratory data have shown that most patients had a decrease in lymphocyte count, Eosinophils count, platelets count, hemoglobin, and albumin level. Also, laboratory data recorded an elevation in Leukocyte, ESR, PT, D-dimer, PCT, CRP, ALT, AST, Bilirubin, Creatinine, CK, LDH, Ferritin, Troponin, Myoglobin, IL-6, IL10 and TNF.

- The level of Lymphocyte count, Eosinophil count, LDH, CRP can be used in the detection of COVID-19 patients.

- It is recommend that using chest X-ray along with the selected available parameters: lymphocyte count, eosinophil count, CRP and LDH as routine tests for the initial identification of suspected cases of COVID-19 till the result of RT-PCR is revealed.

\section{REFERENCE}

[1] World Health Organization, "WHO Timeline - COVID-19.” [Online]. Available: https://www.who.int/newsroom/detail/27-04-2020-who-timeline---covid-19. [Accessed: 29-May-2020].

[2] A. E. Gorbalenya et al., "The species Severe acute respiratory syndrome-related coronavirus: classifying 2019-nCoV and naming it SARS-CoV-2,” Nat. Microbiol., vol. 5, no. 4, pp. 536-544, 2020.

[3] World Health Organization, "Naming the coronavirus disease (COVID-19) and the virus that causes it." [Online]. Available: https://www.who.int/emergencies/diseases/novel-coronavirus-2019/technicalguidance/naming-the-coronavirus-disease-(covid-2019)-and-the-virus-that-causes-it. [Accessed: 29-May2020].

[4] N. Petrosillo, G. Viceconte, O. Ergonul, G. Ippolito, and E. Petersen, "COVID-19, SARS and MERS: are they closely related?," Clin. Microbiol. Infect., vol. 26, no. 6, pp. 729-734, 2020.

[5] Iraq ministry of health, “الموقف الوبائي اليومي للإصنابات بفيروس... - وزارة الصحة العراقية." [Online]. Available: https://www.facebook.com/MOH.GOV.IQ/photos/a.860171854037214/2840429512678095/?type=3. [Accessed: 29-May-2020].

[6] Kurdistan regional government, "COVID-19: Situation Update - GOV.KRD." [Online]. Available: https://gov.krd/coronavirus-en/situation-update/. [Accessed: 29-May-2020].

[7] Z. Chen et al., "From SARS-CoV to Wuhan 2019-nCoV: Will History Repeat Itself?," bioRxiv. pp. 1-18, 2020 .

[8] J. F. W. Chan et al., "A familial cluster of pneumonia associated with the 2019 novel coronavirus indicating person-to-person transmission: a study of a family cluster," Lancet, vol. 395, no. 10223, pp. 514-523, 2020.

[9] Q. Li et al., "Early transmission dynamics in Wuhan, China, of novel coronavirus-infected pneumonia," N. Engl. J. Med., vol. 382, no. 13, pp. 1199-1207, 2020.

[10] World Health Organization, "Laboratory testing for 2019 novel coronavirus (2019-nCoV) in suspected human cases." [Online]. Available: https://www.who.int/publications-detail/laboratory-testing-for-2019-novelcoronavirus-in-suspected-human-cases-20200117. [Accessed: 29-May-2020].

[11] T. Ai et al., "Correlation of Chest CT and RT-PCR Testing in Coronavirus Disease 2019 (COVID-19) in China: A Report of 1014 Cases," Radiology, 2020.

[12] X. Xie, Z. Zhong, W. Zhao, C. Zheng, F. Wang, and J. Liu, "Chest CT for Typical 2019-nCoV Pneumonia: Relationship to Negative RT-PCR Testing," Radiology, 2020.

[13] D. Li et al., "False-negative results of real-time reverse-transcriptase polymerase chain reaction for severe acute respiratory syndrome coronavirus 2: Role of deep-learning-based ct diagnosis and insights from two cases," Korean J. Radiol., vol. 21, no. 4, pp. 505-508, 2020.

[14] B. Cao, "Chinese Guideline of Diagnosis and treatment of COVID-19 Severe Acute Respiratory Syndrome Coronavirus-2.” pp. 1-30, 2020.

[15] Novel Coronavirus Pneumonia Emergency Response Epidemiology Team chinese center for disease control and prevention, "Vital surveillances: the epidemiological characteristics of an outbreak of 2019 novel coronavirus diseases (COVID-19)—China, 2020," China CDC Wkly., vol. 41, no. 2, pp. 145-151, 2020.

[16] A. J. Rodriguez-Morales et al., "Clinical, laboratory and imaging features of COVID-19: A systematic review and meta-analysis,” Travel Med. Infect. Dis., vol. 34, pp. 1-13, 2020.

[17] G. Lippi and M. Plebani, "Laboratory abnormalities in patients with COVID-2019 infection," Clinical Chemistry and Laboratory Medicine. pp. 1-4, 2020.

[18] L. Fu et al., "Clinical characteristics of coronavirus disease 2019 (COVID-19) in China: A systematic review and meta-analysis," J. Infect., vol. 80, no. 6, pp. 656-665, 2020.

[19] W. Guan et al., "Clinical characteristics of coronavirus disease 2019 in China,” N. Engl. J. Med., vol. 382 , no. 18, pp. 1708-1720, 2020. 
[20] C. Huang et al., "Clinical features of patients infected with 2019 novel coronavirus in Wuhan, China," Lancet, vol. 395 , no. 10223 , pp. 497-506, 2020.

[21] L. Tan et al., "Lymphopenia predicts disease severity of COVID-19: a descriptive and predictive study," Signal Transduct. Target. Ther., vol. 5, no. 33, pp. 1-3, 2020.

[22] Q. Li et al., "A simple laboratory parameter facilitates early identification of COVID-19 patients," medRxiv. pp. 1-6, 2020.

[23] J. jin Zhang et al., "Clinical characteristics of 140 patients infected with SARS-CoV-2 in Wuhan, China," Allergy: European Journal of Allergy and Clinical Immunology. pp. 1-12, 2020.

[24] Z. W. Weiliang Cao, Li Shi, Lin Chen, Xuemei Xu, "Clinical features and laboratory inspection of novel coronavirus pneumonia (COVID-19) in Xiangyang, Hubei," medRxiv. pp. 1-13, 2020.

[25] D. Wang et al., "Clinical Characteristics of 138 Hospitalized Patients with 2019 Novel Coronavirus-Infected Pneumonia in Wuhan, China,” JAMA - J. Am. Med. Assoc., vol. 323, no. 11, pp. 1061-1069, 2020.

[26] N. Chen et al., "Epidemiological and clinical characteristics of 99 cases of 2019 novel coronavirus pneumonia in Wuhan, China: a descriptive study," Lancet, vol. 395, no. 10223, pp. 507-513, 2020.

[27] J. Zhang, L. Zhou, Y. Yang, W. Peng, W. Wang, and X. Chen, "Therapeutic and triage strategies for 2019 novel coronavirus disease in fever clinics," Lancet Respir. Med., vol. 8, no. 3, pp. e1-e2, 2020.

[28] F. A. Lagunas-Rangel, "Neutrophil-to-lymphocyte ratio and lymphocyte-to-C-reactive protein ratio in patients with severe coronavirus disease 2019 (COVID-19): A meta-analysis," Journal of Medical Virology. pp. 1-2, 2020

[29] Y. Liu et al., "Clinical and biochemical indexes from 2019-nCoV infected patients linked to viral loads and lung injury,” Sci. China Life Sci., vol. 63, no. 3, pp. 364-374, 2020.

[30] A. Bastola et al., "The first 2019 novel coronavirus case in Nepal," Lancet Infect. Dis., vol. 20, no. 3, pp. 279-280, 2020.

[31] G. Spiteri et al., "First cases of coronavirus disease 2019 (COVID-19) in the WHO European Region, 24 January to 21 February 2020," Eurosurveillance, vol. 25, no. 9, pp. 1-6, 2020.

[32] W. K. Silverstein, L. Stroud, G. E. Cleghorn, and J. A. Leis, "First imported case of 2019 novel coronavirus in Canada, presenting as mild pneumonia," Lancet, vol. 395, no. 10223, p. 734, 2020.

[33] S. A. Kujawski et al., "Clinical and virologic characteristics of the first 12 patients with coronavirus disease 2019 (COVID-19) in the United States," Nature Medicine. pp. 1-23, 2020.

[34] R. Mardani et al., "Laboratory Parameters in Detection of COVID-19 Patients with Positive RT-PCR; a Diagnostic Accuracy Study.," Arch. Acad. Emerg. Med., vol. 8, no. 1, p. e43, 2020.

[35] A. Ahmed Hama, O. Abdulrahman Mohammed, F. Mahmud Ali, O. Hamid Shareef, S. Muhammad Wli, and S. Ahmed Qadir, "The Molecular Diagnosis Protocols of New Coronavirus (COVID-19); Specificity and Sensitivity an Overview," Kurdistan J. Appl. Res., vol. 5, no. 3, pp. 13-22, 2020.

[36] World Health Organization, "Coronavirus disease (COVID-19) technical guidance: Laboratory testing for 2019-nCoV in humans." [Online]. Available: https://www.who.int/emergencies/diseases/novel-coronavirus2019/technical-guidance/laboratory-guidance. [Accessed: 29-May-2020].

[37] P. Zhou et al., "A pneumonia outbreak associated with a new coronavirus of probable bat origin," Nature, vol. 579, no. 7798, pp. 270-273, 2020.

[38] S. L. Bai et al., "Analysis of the first cluster of cases in a family of novel coronavirus pneumonia in Gansu Province," Zhonghua Yu Fang Yi Xue Za Zhi, vol. 54, pp. 1-4, 2020.

[39] S. Y. Xiao, Y. Wu, and H. Liu, "Evolving status of the 2019 novel coronavirus infection: Proposal of conventional serologic assays for disease diagnosis and infection monitoring," J. Med. Virol., vol. 92, no. 5, pp. 464-467, 2020.

[40] Centers for Disease Control and Prevention, "Serology Testing for COVID-19 at CDC." [Online]. Available: https://www.cdc.gov/coronavirus/2019-ncov/lab/serology-testing.html. [Accessed: 29-May-2020].

[41] U.S. Food and Drug Administration, "Coronavirus (COVID-19) Update: Serological Tests." [Online]. Available: https://www.fda.gov/news-events/press-announcements/coronavirus-covid-19-update-serologicaltests. [Accessed: 29-May-2020].

[42] Z. Wu and J. M. McGoogan, "Characteristics of and Important Lessons From the Coronavirus Disease 2019 (COVID-19) Outbreak in China,” JAMA, vol. 323, no. 13, pp. 1239-1242, 2020.

[43] X. Xu et al., "Imaging and clinical features of patients with 2019 novel coronavirus SARS-CoV-2," Eur. J. Nucl. Med. Mol. Imaging, vol. 47, no. 5, pp. 1275-1280, 2020.

[44] Y. Fang et al., "Sensitivity of Chest CT for COVID-19: Comparison to RT-PCR," Radiology. pp. 1-8, 2020.

[45] M.-Y. Ng et al., "Imaging Profile of the COVID-19 Infection: Radiologic Findings and Literature Review," Radiol. Cardiothorac. Imaging, vol. 2, no. 1, pp. 1-26, 2020.

[46] H. Y. F. Wong et al., "Frequency and Distribution of Chest Radiographic Findings in COVID-19 Positive Patients," Radiology. pp. 1-23, 2019.

[47] Centers for Disease Control and Prevention, "CDC 2019-Novel Coronavirus (2019-nCoV) Real-Time RTPCR Diagnostic Panel.” pp. 1-53, 2020.

[48] D. Ferrari, A. Motta, M. Strollo, G. Banfi, and M. Locatelli, "Routine blood tests as a potential diagnostic tool for COVID-19," Clinical Chemistry and Laboratory Medicine. pp. 1-5, 2020. 